\title{
A SWEET solution to rice blight
}

Two studies offer a powerful strategy for combating bacterial blight in rice.

Rajeev K. Varshney, Ian D. Godwin, Trilochan Mohapatra, Jonathan D. G. Jones and Susan R. McCouch

B acterial blight is an important disease of rice that is particularly destructive in Southeast Asia and sub-Saharan Africa, exacerbated by the heavy rains of the monsoon seasons. Estimated crop loss due to bacterial blight may be as high as $75 \%$, with millions of hectares of rice affected annually. In this issue, an international team of researchers describes the use of CRISPR editing to generate rice plants that are broadly resistant to the main pathogen that causes rice blight, Xanthomonas oryzae pv. oryzae $(\mathrm{Xoo})^{1}$. To enhance the durability and management of resistance, the team has also developed a kit to trace the disease, and its virulence and resistance alleles ${ }^{2}$.

The most sustainable, cost-effective and safe approach to controlling rice blight is the use of genetically resistant plants. A total of 43 different genes conferring host resistance to bacterial blight have been identified in rice so far, and a subset has been characterized ${ }^{3}$. Through genomicsassisted breeding, more than ten resistant rice varieties have been developed. Several of these varieties ${ }^{4}$, which rely on resistance genes Xa4, xa5, xa13, Xa21, Xa33 and Xa38, have been released for commercial cultivation across the globe.

In the biological arms race that is plantpathogen interactions, plant resistance is often overcome by the emergence of potent pathogen virulence mechanisms. The Xoo pathogen produces specific effector molecules that promote the host's provision of the sugar required for bacterial growth. These transcription activator-like effectors (TALEs) bind to effector-binding elements (EBEs) in the promoters of SWEET genes
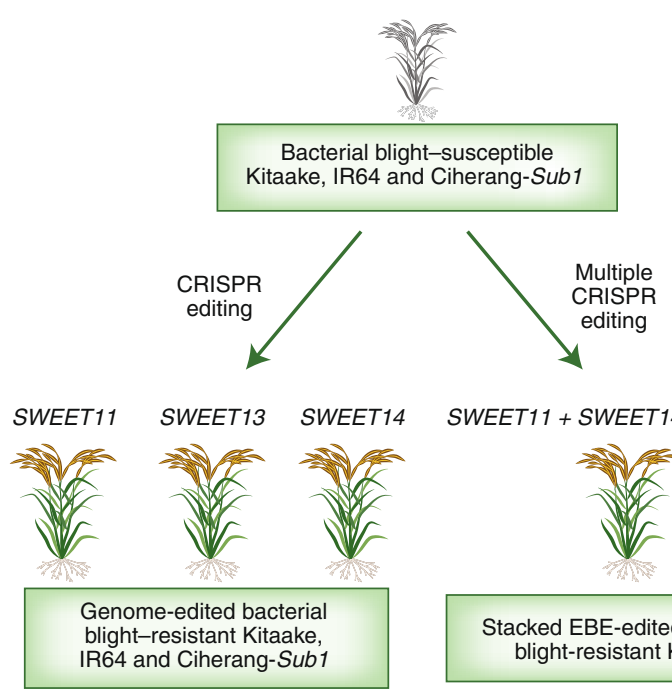

SWEET11 + SWEET14 + SWEET13

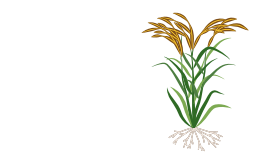

Stacked EBE-edited bacterial blight-resistant Kitaake

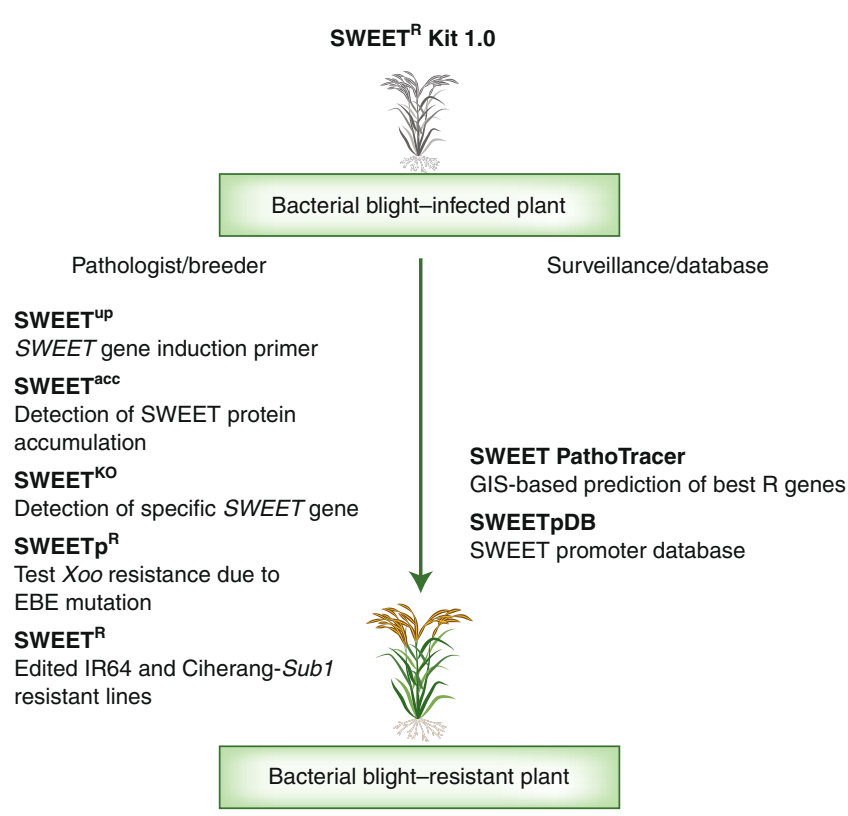

Bacterial blight-resistant plant

Fig. 1 | Overview of the development of genome-edited lines for key SWEET genes and features of a diagnostic kit for combating bacterial blight rice. a, Development of genome-edited lines for three (SWEET 11, SWEET13 and SWEET14) genes in the genetic background of Kitaake, IR64 and Ciherang-Sub1. Edited EBEs for these genes were also stacked in the genetic background of Kitaake. Phenotyping of the lines showed full resistance to bacterial blight with the current set of Xoo strains. Therefore, after ensuring that they do not contain off-target mutations and confirming acceptable agronomic performance in mega environments, these lines can be deployed in rice-growing countries that allow genome-edited products. $\mathbf{b}$, Components of a diagnostic kit for combating bacterial blight in rice. The kit includes SWEETup, a qRT-PCR primer set for detection of SWEET gene induction by Xoo strain; SWEETacc, GUS tester lines in the background of Kitaake for the detection of SWEET protein accumulation by Xoo strains; SWEET ${ }^{\text {ko }}$, knockout tester lines in the background of Kitaake for the detection of specific SWEET gene dependences; SWEETp ${ }^{R}$, tester lines in the background of Kitaake to study whether EBE mutations cause Xoo resistance; and SWEET ${ }^{R}$, genome edited rice lines in the background of IR64 and Ciherang-Sub1 containing multiple SWEET promoter variants. The kit also contains SWEETPDB, a SWEET promoter database for the identification of TALE targets in different rice lines, and a web-based SWEET PathoTracer for geographical information system-based prediction of optimal resistance gene deployment. This kit can be used to analyze pathogen populations in the field in a given geographic region and then to identify suitable resistant lines from the kit. These lines can be used as resistant donors, and desirable resistant lines can be developed through molecular breeding approaches. 
(a 21-gene family in rice), which encode sugar transporters. Activation of target SWEET genes elevates sugar transport to where the pathogen needs it, enhancing its virulence and leading to disease susceptibility.

Oliva et al. ${ }^{1}$ undertook an extensive survey of Xoo TALE diversity in 33 Asian and 30 African isolates, sequencing 856 distinct TALEs. They found more copies of TALEs in Asian isolates (18-21 per genome) than in African isolates ( 9 per genome). The high number of TALEs indicated that the challenge of creating a TALE-insensitive line was greater than previously realized. The authors rose to this challenge by using CRISPR-Cas9 to edit promoters of SWEET11, SWEET13 and SWEET14 at EBEs recognized by pathogen TALEs with the aim of recovering SWEET promoter alleles of all three genes with reduced TALE binding.

TALE nuclease-directed mutation of SWEET14 was reported earlier for the development of bacterial blight-resistant lines to specific Xoo strains ${ }^{5,6}$. However, at that time, relatively few Xoo strains had been assessed for TALE gene content. Recently, natural variation in the promoters of SWEET13 and SWEET14 discovered in the $3 \mathrm{~K}$ rice genome sequences ${ }^{7}$ expanded the range of known variants conferring Xoo resistance . $^{8}$

Oliva et al. ${ }^{1}$ chose to target specific EBE polymorphisms based on their sequencelevel characterization of TALEs in $63 \mathrm{Xoo}$ strains from diverse areas, and the mutations they discovered in the EBEs in promoters of SWEET11, SWEET13 and SWEET14 in rice. First, they engineered a rapidly cycling japonica line, Kitaake. To facilitate breeding in South East Asian programs, they then generated the same edits in two mega indica rice varieties, IR64 and Ciherang-Sub1 (Fig. 1a).

Twenty-one SWEET variants in the mega varieties showed high levels of resistance to tested Xoo strains of bacterial blight. Given that the SWEET genes have key roles in sugar transport and signaling in the developing plant, it was reassuring that no adverse effects on field performance, plant development or grain yield were observed. Furthermore, they applied a multiplexed genome editing approach to systematically interfere with SWEET gene induction at all known major TALE EBEs, and engineered
Kitaake lines resistant to all currently known Xoo strains (Fig. 1a). This strategy builds on a deep understanding of the mechanisms governing host-pathogen interaction, and we anticipate that it will be applied to develop broad-spectrum disease resistance in other crops.

In the companion paper, Eom et al. ${ }^{2}$ developed a diagnostic kit, called SWEET ${ }^{\mathrm{R}}$ $\mathrm{v} 1.0$, for analyzing field strains of the rice bacterial blight pathogen, for predicting SWEET genes targeted by Xoo TAL effectors and for customizing the introduction of resistant variants into elite rice varieties to manage the disease. The toolset includes a SWEET promotor database (SWEETpDB); SWEET ${ }^{\mathrm{up}}$ qRT-PCR primers; three SWEET ${ }^{\text {acc }}$ rice tester lines; single and combined SWEET ${ }^{\mathrm{ko}}$ knock-out mutants; SWEETp $^{\mathrm{R}}$ tester lines in the R-gene-free Kitaake background; a web-based SWEET PathoTracer decision tool; and 32 transgene free EBE-edited lines of the indica mega varieties, IR64 and Ciherang-Sub1. The kit is designed to facilitate systematic genomic analysis of Xoo populations, evaluate the efficacy of resistant tester lines, and accelerate development and deployment of bacterial blight-resistant rice lines, which can be released alone or in multi-line combinations (Fig. 1b). A similar concept can be used to manage disease in other crops, like cotton and cassava, that show SWEET-based resistance to bacterial blight.

In our view, these two papers provide a compelling strategy for combating bacterial blight in rice. However, as noted by Oliva et al. ${ }^{1}$, whole-genome sequencing of genome-edited lines is required to identify possible off-target mutations, and field testing in different environments for bacterial blight and agronomic trait performance is needed before formal release and deployment.

Genome editing has now been used to generate useful traits in many crops-such as rice, maize, wheat, sugarcane, soybean, potato, sorghum, orange, cucumber, tomato, flax and cassava-targeting herbicide resistance, drought-stress tolerance, thermosensitive genic male sterility, disease resistance and altered product quality, with some in the process of release for commercial cultivation ${ }^{9}$. The stewardship of gene-edited lines in combination with an appropriate deployment strategy is essential to meet environmental health and safety standards. There remains a lack of clarity as to the non-GMO status of such germplasm in many countries worldwide ${ }^{10}$. We hope that legislation and public opinion will soon allow the benefits of this research to reach the farming community.

Furthermore, we note that the diagnostic kit for the development of blight-resistant rice, together with the genome-edited SWEET R-lines, will be useful in tracking the evolution of the pathogen and selecting suitable donors carrying desirable resistance genes that can be introduced into new varieties through genomic-assisted breeding approaches (Fig. 1). We are confident that these studies will be helpful for many other crops in which genome sequencing for largescale germplasm collections is underway, and a similar strategy can be used to develop genome-edited lines and kits to help manage both current and future diseases.

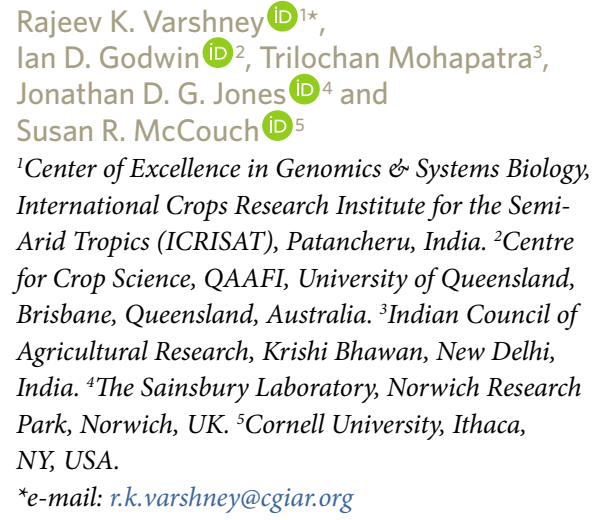

${ }^{1}$ Center of Excellence in Genomics \& Systems Biology, International Crops Research Institute for the SemiArid Tropics (ICRISAT), Patancheru, India. ${ }^{2}$ Centre for Crop Science, QAAFI, University of Queensland, Brisbane, Queensland, Australia. ${ }^{3}$ Indian Council of Agricultural Research, Krishi Bhawan, New Delhi, India. ${ }^{4}$ The Sainsbury Laboratory, Norwich Research Park, Norwich, UK. ${ }^{5}$ Cornell University, Ithaca, NY, USA.

*e-mail:r.k.varshney@cgiar.org

Published online: 28 October 2019

https://doi.org/10.1038/s41587-019-0302-0

References

1. Oliva, R. et al. Nat. Biotechnol. https://doi.org/10.1038/s41587019-0267-z (2019)

2. Eom, J. S. et al. Nat. Biotechnol. https://doi.org/10.1038/s41587019-0268-y (2019).

3. Kim, S. M. \& Reinke, R. F. PLoS One 14, e0211775 (2019).

4. Chukwu, S. C. et al. Mol. Biol. Rep. 46, 1519-1532 (2019).

5. Li, T., Liu, B., Spalding, M. N., Weeks, D. P. \& Yang, B. Nat. Biotechnol. 30, 390-392 (2012).

6. Blanvillain-Baufumé, S. et al. Plant Biotechnol. J. 15, 306-317 (2017).

7. Wang, W. et al. Nature 557, 43-49 (2018).

8. Zaka, A. et al. PLoS One 13, e0203711 (2018).

9. Zhang, Y., Massel, K., Godwin, I. D. \& Gao, C. Genome Biol. 19, 210 (2018).

10. Schulman, A. H., Oksman-Caldentey, K. M. \& Teeri, T. H. Plant Biotechnol. J. https://doi.org/10.1111/pbi.13200 (2019).

Competing interests

The authors declare no competing interests. 